\title{
TROMBOEMBOLISMO PULMONAR: VENOGRAFIA POR TOMOGRAFIA COMPUTADA VERSUS ECOGRAFIA DOPPLER, PARA DETECCION DE TROMBOSIS VENOSA PROFUNDA DE EXTREMIDADES INFERIORES
}

\section{Drs. Gonzalo Miranda $G^{(1)}$, Juan Carlos Díaz $P^{(1)}$, Patricia Arancibia $H^{(1)}$, Internos Carolina Díaz $G^{(2)}$, Alvaro Vidal $F^{(2)}$.}

1. Centro de Imagenología. Hospital Clínico de la Universidad de Chile.

2. Facultad de Medicina. Universidad de Chile. Hospital San Juan de Dios.

\begin{abstract}
Pulmonary embolism is a condition with high morbidity and mortality. The deep venous thrombosis at the lower limbs remains as the main cause, so the evaluation of this venous system must be ruled out in patients at risk. Doppler US as CT venography of the lower limbs are the most useful diagnostic methods. The present study compares Doppler venous US findings with CT venography in the diagnostic of deep venous thrombosis in patients that were clinically suspected. It was found a fairly good correlation between them, sheering high sensibility and specificity
\end{abstract}

Key words: CT venography, Deep venous thrombosis, Pulmonary embolism.

Resumen: El tromboembolismo pulmonar (TEP) es un cuadro de alta morbimortalidad. La principal fuente embolígena, es la trombosis venosa profunda (TVP) de las extremidades inferiores (EEII), por ello, el estudio de un paciente con sospecha clínica de TEP, incluye la evaluación de estas venas. La venografía por tomografía computada (TC) ha demostrado utilidad en la evaluación de la TVP, existiendo numerosos trabajos que avalan su buena correlación con el Doppler venoso de EEll. Nuestro estudio compara ambos métodos en la detección de TVP de las EEII en estos pacientes.

Encontramos una muy buena correlación

Miranda G, y cols. Tromboembolismo pulmonar: Venografía por tomografía computada versus ecografía Doppler, para detección de trombosis venosa profunda de extremidades inferiores. Rev Chil Radiol 2005; 11:5-8.

Correspondencia: Dr. Gonzalo Miranda González.

Hospital Clínico de la Universidad de Chile.

Santos Dumont 999. Independencia.

gomiranda2000@yahoo.com entre ambos métodos, con una sensibilidad del 100\% y una especificidad de $88,9 \%$ para la venografía por TC comparado con el Doppler, lo que concuerda con lo descrito en la literatura.

Palabras claves: Tromboembolismo pulmonar, Trombosis venosa profunda, Venografía por TC.

\section{Introducción}

A pesar de los avances diagnósticos y terapéuticos, el tromboembolismo pulmonar (TEP) sigue siendo una complicación severa y potencialmente fatal de cuadros médicos y quirúrgicos, llegando a una cifra de más de 200.000 muertes en EE.UU. cada año, por esta causa ${ }^{(4)}$. EI origen más frecuente es la trombosis venosa, que en más del $90 \%$ de los casos se origina en las venas profundas de las extremidades inferiores.

EI TEP no presenta un cuadro clínico característico, por lo que constituye una patología de difícil diagnóstico. Dado que su tratamiento precoz disminuye su mortalidad ${ }^{(1)}$, es de real importancia un diagnostico rápido y la pesquisa precoz de factores predisponentes.

La tomografía computada (TC) helicoidal, especialmente la modalidad multicorte, ha llegado a ser una de las mejores herramientas en el diagnóstico de TEP(3), aun cuando en algunas determinadas situaciones algunos utilizan aún la cintigrafía ventilación-perfusión ${ }^{(5)}$. En la mayoría de los centros se prefiere el estudio tomográfico por su gran exactitud en el diagnóstico de trombosis pulmonar central, otorgando además, la oportunidad de estudiar el resto de los órganos adyacentes, lo que reviste una gran importancia en el diagnóstico diferencial de un paciente con sintomatología torácica ${ }^{(3)}$.

Debido a la asociación entre TEP y trombosis de las extremidades inferiores (EEII), es que en 
mayoría de los centros, el algoritmo de estudio ante la sospecha de TEP incluye la evaluación de estas últimas. Aunque no existe un estándar de referencia en el diagnóstico de trombosis venosa profunda (TVP) ${ }^{(6)}$, la ultrasonografía Doppler se utiliza ampliamente como el único estudio inicial, dado que presenta una sensibilidad y especificidad del $90 \%$ en la detección de trombosis venosa sobre el nivel de las venas poplítea ${ }^{(4)}$. Es un examen económico, de gran utilidad en pacientes críticos con dificultad para su traslado, además no los expone a los riesgos del medio de contraste y radiación, esto último especialmente importante en pacientes jóvenes ${ }^{(6)}$.

La TC sin embargo, es el único método diagnóstico que permite evaluar en un mismo tiempo los sistemas vascular pulmonar y de las extremidades inferiores, existiendo numerosas publicaciones que avalan su rendimiento en el diagnóstico de $\operatorname{TVP}^{(5,7)}$.

Objetivos: Estudiar la correlación entre los resultados de la ultrasonografía Doppler venoso versus la venografía por TC, en la detección de trombosis venosa profunda de las EEII, en pacientes con sospecha clínica de TEP.

\section{Materiales y métodos}

El estudio fue realizado entre Febrero del 2003 y Marzo del 2004, en un total de 52 pacientes internados en el Hospital Clínico de la Universidad de Chile y quienes fueron derivados por sospecha clínica de TEP. Se les realizó angiografía pulmonar y venografía de EEIl por TC y además ecografía Doppler venoso de extremidades. Entre ambos exámenes hubo un intervalo de tiempo máximo de 7 días.

Para TC el equipo usado fue un Siemens Emotion Duo. En la angio-TC pulmonar se realizó adquisición helicoidal desde los vértices pulmonares hasta los recesos costofrénicos posteriores, con cortes de $3 \mathrm{~mm}$, administrándose $150 \mathrm{ml}$ de medio de contraste hidrosoluble, que en nuestro caso fue Optiray $(320 \mathrm{mg} / \mathrm{l})$, a una velocidad de infusión de 3 $\mathrm{ml} / \mathrm{seg}$. El protocolo para búsqueda de TVP se realizó mediante adquisición secuencial desde el tercio distal de la vena cava inferior hasta el extremo distal de las venas poplíteas, con cortes de $10 \mathrm{~mm}$ de espesor e intervalo de $10 \mathrm{~mm}$ consecutivos, con un delay de 3 minutos a contar del término de la adquisición en tórax la que dura en promedio $30 \mathrm{seg}$. Se utilizó el algoritmo de reconstrucción de partes blandas (B30s). Cuando fue necesario se realizaron además reconstrucciones multiplanares. Los estudios angiográficos pulmonares y venografías por TC fueron informados por un radiólogo con experiencia en imágenes torácicas (JCD) y un residente de tercer año (GM), quienes desconocían el resultado de cualquier otro examen previo. La lectura fue efectuada a partir de las placas impresas y de las imágenes correspondientes visualizadas en el monitor del sistema digital IMPAX.
Los criterios de diagnóstico de TVP fueron la visualización de un defecto de llene endoluminal y/o la no opacificación de un segmento venoso.

El estudio Doppler venoso de extremidades inferiores se realizó en equipos ATL 5000, Ultramark 9 y Siemens Antares, por radiólogos con distinto grado de experiencia. El examen se extendió desde las venas femorales hasta las venas de las piernas. Los criterios de diagnostico para TVP fueron: la ausencia de compresión venosa, la ocupación del lumen por material ecogénico y la ausencia de señal venosa al Doppler color y espectral.

El análisis de los resultados se realizó a través de medidas de correlación con Kappa por método de Bennett y se obtuvieron sensibilidades, especificidades, valor predictivo positivo y negativo, por método tradicional, usando la ecografía Doppler como el estándar de referencia.

\section{Resultados}

Se demostró trombosis venosa profunda en 13 de los 52 casos estudiados. De ellas en 8 pacientes se ubicaron en el territorio fémoro-poplíteo, explorado por ambos métodos, en 8 pacientes demostrados por TC (Figura 1) y en 7 por ecografía (Figura 2), (tabla I). Los 5 pacientes restantes presentaron trombosis venosa profunda por debajo de las venas poplíteas, territorio explorado sólo por la ecografía Doppler (Figura 3).

La concordancia en el territorio explorado por ambos métodos (fémoro-poplíteo) fue en 7 de 8 casos, lo que nos da una correlación simple de 0,875 con un valor $k$ de 0,92 , que corresponde a una correlación muy buena.

Hubo un falso positivo de la venografía por TC y correspondió a una trombosis de la vena safena interna.

Si se analiza la concordancia entre ambos métodos incluyendo los distintos territorios evaluados, esto es, incluyendo la evaluación de las piernas, la TC detecta 8 de los 13 casos, lo que nos da una correlación simple de 0,61 con un valor Kappa de 0,74 , que corresponde a una correlación buena.

Para el territorio fémoro-poplíteo, la venografía por TC presenta una sensibilidad del $100 \%$ con un intervalo de confianza de 0,64 a 1 y una especificidad del $88,9 \%$ con un intervalo de confianza de 0,76 a 0,95 . El valor predictivo positivo fue de $88 \%$ y el negativo de $100 \%$.

Se comprobó tromboembolismo pulmonar solo en un $32 \%$ de los pacientes con sospecha clínica (17 de 52). De éstos en un 64\% se demostró TVP (11 de 17) (Figura 4).

De los pacientes con TVP, el $100 \%$ de los que presentaron localización fémoro-poplítea tuvieron TEP (8 de 8) y 3 de los 5 pacientes con localización bajo las venas poplíteas también lo tuvieron. 
La venografía por TC mostró trombosis de la vena femoral común en 2 pacientes en que la ecografía mostró sólo trombosis de la vena femoral superficial (Tabla II).

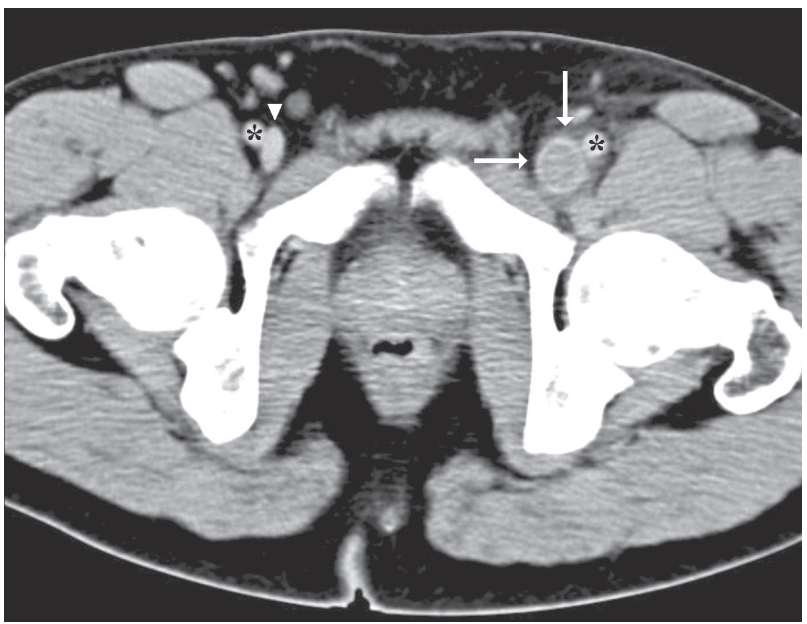

Figura 1. Venografía por TC. Trombosis de la vena femoral común izquierda (flechas). Punta de flecha: Vena femoral común derecha; Asteriscos ( $\left.{ }^{*}\right)$ : Arterias femorales comúnes.

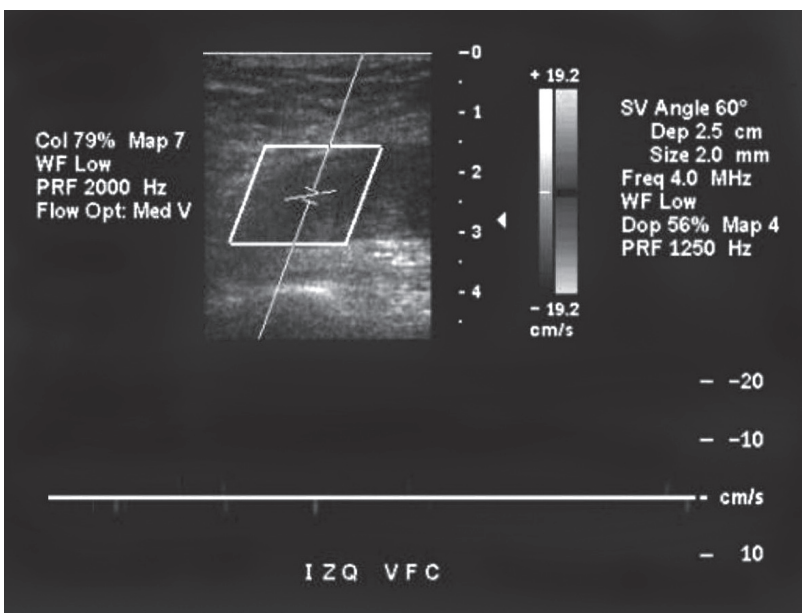

Figura 2. Ecografía demostrando trombosis de la vena femoral común izquierda, la que es no compresible y presenta la imagen clásica de contenido hipoecogenico intraluminal y ausencia de señal al Doppler color y espectral.

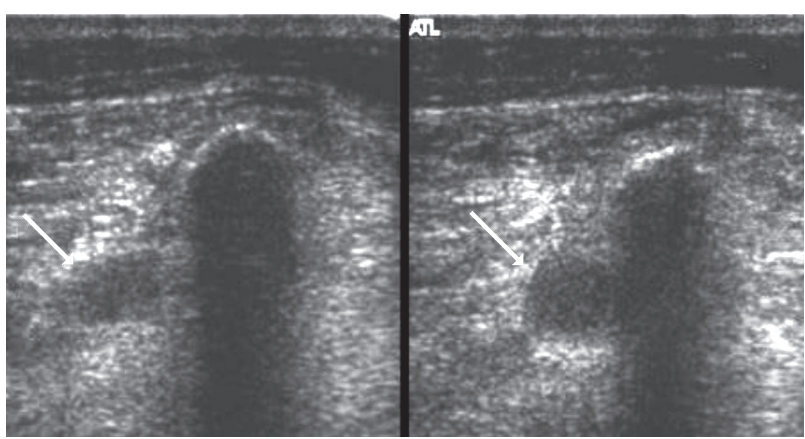

Figura 3. Ecografía en imagen dual, demostrando trombosis de venas de las piernas (flechas). La evaluación de estas venas es controversial, ya que es considerada de bajo riesgo para generar TEP clínicamente significativo o para propagarse hacia proximal.

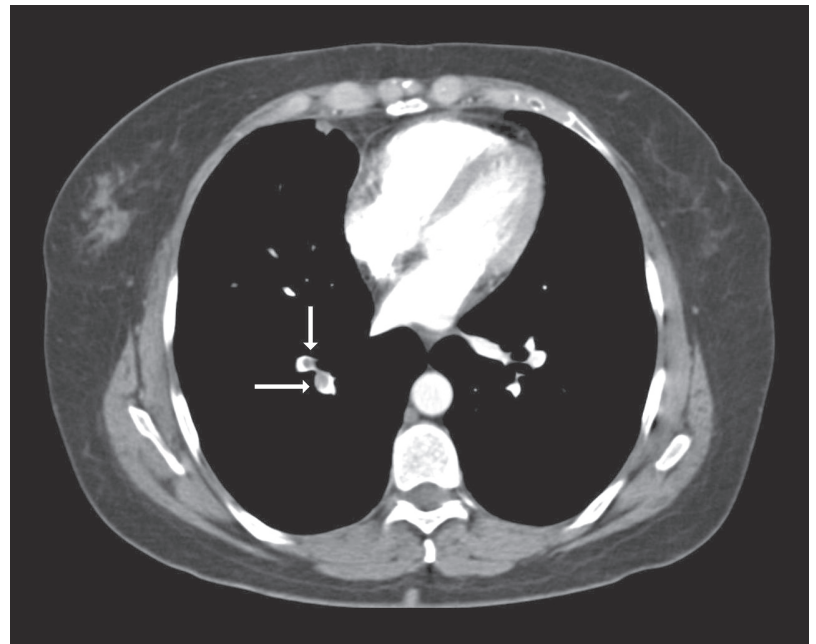

Figura 4. Angio-TC de tórax demostrando TEP manifestado como defectos de llene en ramas segmentarias basales derechas (flechas).

Tabla I. Correlación de hallazgos en TC vs Doppler (US) en la detección de trombosis venosa profunda femoro-poplítea.

$\begin{array}{llll}\text { US/TC } & (+) & (-) & \text { Total }\end{array}$

\begin{tabular}{lrrr}
\hline$(+)$ & 7 & 1 & 8 \\
$(-)$ & 0 & 44 & 44 \\
Total & 7 & 45 & 52
\end{tabular}

Tabla II. Sitio de trombosis según ambos métodos. Detección de la extensión de la trombosis por US vs. TC

\begin{tabular}{lcc} 
& US & TC \\
\hline Vena poplítea & 1 & 1 \\
Vena femoral superficial & 4 & 2 \\
Vena femoral común & 2 & 4
\end{tabular}

\section{Discusión}

La TC helicoidal es hoy en día uno los mejores métodos diagnósticos para TEP, dado su gran exactitud en el diagnóstico de trombosis central ${ }^{(3)}$. A su vez, es el único método de diagnóstico que permite evaluar en un mismo tiempo los sistemas vascular pulmonar y venoso de las EEll. Garg et al en un estudio realizado en 70 pacientes, describen una sensibilidad del $100 \%$ y una especificidad del $95 \%$ para la venografía por TC de las EEII, comparado con la ultrasonografía en el territorio femoro-poplíteo(5). En otros estudios la sensibilidad oscila entre un 89 y $100 \%$ y la especificidad entre un 92 y $100 \%$ (7).

La venografía por TC presenta algunas 
ventajas sobre la ecografía Doppler, como el hecho de su rapidez y la no operador dependencia. Por lo demás no requiere la administración adicional de medio de contraste, ya que el barrido en EEll se realiza a continuación de la fase arterial pulmonar.

En nuestro estudio obtuvimos una sensibilidad del $100 \%$ y una especificidad del $88.9 \%$ para la venografía por TC en el diagnóstico de TVP de las extremidades inferiores, en el nivel comprendido entre las venas femoral común y poplíteas, con una buena concordancia con el estudio Doppler, lo cual concuerda con otros estudios publicados.

En uno de nuestros pacientes la TC detectó trombosis en la vena safena interna, inmediatamente bajo su cayado. En él se evidenció TEP en la angiografía pulmonar, lo que sugiere que su hallazgo no es intrascendente y probablemente corresponde más bien a un falso negativo de la ecografía Doppler.

Según lo descrito en la literatura, es controversial la evaluación de las EEII bajo las venas poplíteas $^{(8)}$. Gottlieb et al en un estudio retrospectivo, realizado en 283 pacientes, concluyen que no sería necesaria la evaluación de este segmento mediante ultrasonografía debido al bajo riesgo de generar TEP clínicamente significativo o que la trombosis se propague hacia proximal(9).

Nuestro protocolo de TC no incluyó la evaluación de este territorio, el cual solo fue evaluado por ecografía Doppler, resultando positivo para trombosis en cinco de los 52 casos. Es de relevancia mencionar que tres de estos cinco pacientes que presentaron trombosis solamente bajo las venas poplíteas tuvieron TEP en la evaluación pulmonar por TC. Podemos concluir por lo tanto, que más de la mitad de los pacientes con trombosis solo en este sector presentó TEP, y aun cuando, el número de pacientes evaluado en nuestro estudio no nos permite mencionar cifras significativas, sería importante analizar esta variable en un estudio más extenso.

La venografía por TC mostró trombosis de la vena femoral común en dos pacientes en que la ultrasonografía mostró sólo trombosis de la vena femoral superficial. Esto probablemente se deba a la progresión de la trombosis en el intervalo entre la realización de ambos exámenes, ya que en ambos casos la ecografía Doppler se realizó previamente.

En nuestro protocolo de TC para las EEII utilizamos cortes secuenciales de $10 \mathrm{~mm}$ de espesor; destacando que nuestros hallazgos fueron similares a estudios previos que utilizaron cortes de 5 u $8 \mathrm{~mm}^{(3,4,7)}$, por tanto es posible la disminución de radiación al paciente y el consiguiente ahorro en el uso del tubo de rayos $X$.

\section{Conclusiones}

Similar a lo descrito en la literatura, nuestro estudio muestra que la venografía por TC es tan sensible y específica como la ecografía Doppler en la detección de TVP de EEII, con un buen índice de concordancia, en el segmento comprendido entre las venas femoral común y poplítea, con la ventaja de ser un examen fácil de realizar e interpretar y no ser operador dependiente.

A diferencia de lo descrito en la literatura, un porcentaje no despreciable de pacientes con trombosis de las venas distales al territorio poplíteo presentó TEP, por tanto de considerarse la venografía por TC en el estudio de TVP sería de interés evaluar su utilidad en venas distales a las poplíteas.

\section{Bibliografía}

1. Cerón M, Ortega C, Micco S. Tromboembolismo pulmonar: Estudio multicéntrico. Revista de Medicina Interna Concepción, 1997.

2. Armijo S. Cintigrafía de ventilación y perfusión en el manejo del tromboembolismo pulmonar. Rev Med Chile. 2000; 128: 1011-1014.

3. Loud PA, Katz DS, Klippenstein DL, et al. Combined CT Venography and pulmonary angiography in suspected thromboembolic disease: Diagnostic accuracy for deep venous evaluation. Am J Roentgenol AJR 2000; 174: 61-65.

4. Peterson DA, Kazerooni A, Wakefield TW et al. Computed tomographic venography is specific but not sensitive for diagnosis of acute lower extremity deep venous thrombosis in patients with suspected pulmonary embolus. J Vasc Surg 2001; 34: 798-804.

5. Garg K, Kemp JL, Wojick D, et al. Thromboembolic disease: Comparison of combined CT pulmonary angiography and venography with bilateral leg sonography in 70 Patients. Am J Roentgenol AJR 2000; 175: 997-1001.

6. Maki DD, Kumar N, Nguyen B, et al. Distribution of thrombi in acute lower extremity deep venous thrombosis: Implications for sonography and CT and MR venography. Am J Roentgenol AJR 2000; 175: 1299-1301.

7. Begemann PG, Bonacker M, Kemper J, et al. Evaluation of the deep venous system in patients with suspected pulmonary embolism with multi-detector CT: A prospective study in comparison to Doppler sonography. J Comput Assist Tomogr. 2003, 27: 399-409.

8. Cronan JJ. Venous thromboembolic disease: the role of US. Radiology. 1993; 186: 619-630.

9. Gottlieb RH, Widjaja J, Mehra S, Robinette WB. Clinically Important pulmonary emboli: Does calf vein US alter outcomes? Radiology 1999; 211: 25-29.

10. Duwe KM, Shiau M, Budorick N, et al. Evaluation of the lower extremity veins in patients with suspected pulmonary embolism: A retrospective comparison of helical CT venography and sonography. Am J Roentgenol AJR 2000; 175: 1525-1531.

11. Perrier A, Howarth N, Didier D, et al. Performance of helical computed tomography in unselected outpatients with suspected pulmonary embolism. Ann Intern Med. 2001; 135: 88-97. 